University of Nebraska - Lincoln

DigitalCommons@University of Nebraska - Lincoln

Sociology Department, Faculty Publications

Sociology, Department of

2006

\title{
Mental Disorders among Parents/Caretakers of American Indian Early Adolescents in the Northern Midwest
}

\author{
Les B. Whitbeck \\ University of Nebraska-Lincoln, Iwhitbeck2@unl.edu \\ Dan R. Hoyt \\ University of Nebraska-Lincoln, dhoyt2@unl.edu \\ Kurt Johnson \\ University of Nebraska-Lincoln \\ Xiaojin Chen \\ Tulane University
}

Follow this and additional works at: https://digitalcommons.unl.edu/sociologyfacpub

Part of the Sociology Commons

Whitbeck, Les B.; Hoyt, Dan R.; Johnson, Kurt; and Chen, Xiaojin, "Mental Disorders among Parents/ Caretakers of American Indian Early Adolescents in the Northern Midwest" (2006). Sociology Department, Faculty Publications. 30.

https://digitalcommons.unl.edu/sociologyfacpub/30

This Article is brought to you for free and open access by the Sociology, Department of at DigitalCommons@University of Nebraska - Lincoln. It has been accepted for inclusion in Sociology Department, Faculty Publications by an authorized administrator of DigitalCommons@University of Nebraska - Lincoln. 
Published in Social Psychiatry and Psychiatric Epidemiology 41 (2006), pp. 632-640; doi 10.1007/s00127-006-0070-2 Copyright (C 2006 Steinkopff Verlag, Darmstadt/Springer Verlag. Used by permission. http://www.springerlink.com/link.asp?id=101494

This research was funded by the National Institute on Drug Abuse (DA13580) and the National Institute of Mental Health (MH67281), Les B. Whitbeck, Principal Investigator. The authors acknowledge the helpful comments of two anonymous reviewers for SPPE.

Accepted April 10, 2006; published online June 15, 2006.

\title{
Mental Disorders among Parents/Caretakers of American Indian Early Adolescents in the Northern Midwest
}

\author{
Les. B. Whitbeck, ${ }^{1}$ Dan Hoyt, ${ }^{1}$ Kurt Johnson, ${ }^{1}$ and Xiaojin Chen ${ }^{2}$ \\ ${ }^{1}$ Department of Sociology, University of Nebraska-Lincoln, 739 Oldfather Hall, Lincoln, NE 68588-0324, USA \\ ${ }^{2}$ Department of Sociology, Tulane University, New Orleans, LA, USA \\ Corresponding author - L. B. Whitbeck, lwhitbeck2@unlnotes.unl.edu
}

\begin{abstract}
Background: This study reports prevalence and comorbidty of five DSM-III-R diagnoses (alcohol abuse, alcohol dependence, drug abuse, major depressive episode, and generalized anxiety disorder) among American Indian and Canadian First Nations parents/caretakers of children aged 10-12 years from the Northern Midwest United States and Canada. Lifetime prevalence rates were compared to adults in the National Comorbidity Survey (NCS) and Southwest and Northern Plains cultures from the AI-SUPERPFP study.

Method: Native interviewers used computer-assisted personal interviews to administer the University of Michigan Composite International Diagnostic Interview (UM-CIDI) to 861 tribally enrolled parents and caretakers (625 females; 236 males) of 741 tribally enrolled children aged 10-12 years. Fathers/male caretakers ranged in age from 21 years to 68 years with an average age of 41 years; mothers/female caretakers ranged in age from 17 years to 77 years with an average of 39 years.

Results: About three-fourths $(74.6 \%)$ of the adults met lifetime criteria for one of the five disorders; approximately one-third $(31.6 \%)$ met lifetime criteria for two or more of the five disorders. Prevalence of the substance use disorders was higher than those in the general population (NCS); prevalence of internalizing disorders (major depressive disorder and generalized anxiety disorder) was very similar to those in the general population. Prevalence rates for alcohol abuse among the Northern Midwest adults were higher than those reported for Southwest and Northern Plains Tribes, but rates of alcohol dependency were very similar across cultures.

Conclusions: The higher prevalence rates for some mental disorders found for the Northern Midwest are discussed in terms of potential method variance. The Northern Midwest results reflect unique patterns of psychiatric disorders in the ubiquity of substance abuse disorders and the co-occurrence of substance abuse disorders with internalizing disorders. Reducing lifetime occurrences of substance abuse disorders would have an enormous positive impact on the mental health of this population.
\end{abstract}

Keywords: mental disorder, American Indian

\section{Introduction}

There are numerous barriers to researching mental disorders among Native people in North America. Among the most critical challenges are small, widely-separated, often rural populations in 279 state and federal reservations [1] and major cultural differences between 562 federally recognized tribes in the United States [2]. Geographical isolation and small culturally distinct pop- ulations become an even greater problem when remote Canadian First Nations reserves are part of the culture. These factors create sampling problems that make generalizing to Native people as a group nearly impossible. This diversity has resulted in systematic variations in prevalence of psychiatric disorders between cultural groups [3].

Beals and colleagues in their discussion of the challenge of comparability versus cultural specificity in do- 
ing research on mental disorders point out that in lieu of population-based samples such as their American Indian Service Utilization, Psychiatric Epidemiology, Risk and Protective Factors Project (AI-SUPERPFP), small individual studies that focus on particular cultures contribute to cumulative knowledge regarding mental disorders among American Indian people, particularly if the studies use similar diagnostic measures [3]. However, methodological variations such as community versus specialized samples $[4,5]$ have resulted in wide variation in prevalence estimates. It is often difficult to sort out what is actual variance across cultures and variance attributable to different methodological approaches.

This study uses the same or closely similar cultural modifications of the University of Michigan-Composite International Diagnostic Interview (UM-CIDI) used in the AI-SUPERPFP study of U.S. Southwest and Northern Plains cultures to investigate lifetime and 12-month prevalence of five mental disorders among 861 American Indian and Canadian First Nation parents/caretakers of children aged 10-12 years in the Northern Midwest and Ontario, Canada [6-8]. Although not examining as many disorders as the AI-SUPERPFP, it provides diagnostic information from another Native culture that is comparable to the AI-SUPERPFP adults.

\section{Studies of mental disorder among American Indian adults}

There have been few psychiatric diagnostic studies of Native people and even fewer that use the same diagnostic measures. Studies of psychiatric prevalence have tended to focus on one cultural group $[9,10]$, a specific age group [11-13], a single gender [4] or a specific diagnosis such as alcohol abuse and dependence [14-16] or posttraumatic stress disorder [17]. Due to the lack of representative research, policies and funding decisions often have been based on service utilization studies rather than epidemiological information [18-20].

The first publications from the AI-SUPERPFP data are radically changing the landscape of American Indian psychiatric epidemiology by providing the first population sample that can be compared to national psychiatric epidemiological surveys [5,21]. This important study will become the benchmark for future research on psychiatric disorders in American Indian populations, however, it is limited to two cultures, one Southwest and two very similar Northern Plains tribes. Researchers are now in a position to replicate AI-SUPERPFP work with other Native cultures to provide cumulative and comparable information that will inform policy makers and service providers regarding potential systematic differences in prevalence rates across cultures.

The research reported here benefited from the extensive measurement development of the AI-SUPERPFP team for diagnostic measures. By using simi- lar measures it extends the AI-SUPERPFP research by comparing adults from a Native culture in the Northern Midwest and Ontario, Canada across five psychiatric diagnoses. This research also offers comparisons between "remote" Canadian reserves and rural United States reservations.

\section{Methods}

Sample

These data were collected as part of a 3-year lagged sequential study currently underway on four American Indian reservations in the Northern Midwest and five Canadian First Nation reserves. Four of the Canadian Reserves are classified as "remote" in that they are at considerable distances from even small towns and are accessed by non-paved roads, by boat, over ice in winter, or by airplane. The data are from wave one of the study collected on two U.S. reservations and one Canadian Reserve from February through October, 2002 and wave one of the second group of two U.S. reservations and the four "remote" Canadian reserves collected February through October 2003. The reserves and reservations share a common cultural tradition and language with minor regional variations in dialects. The sample represents one the most populous Native cultures in the United States and Canada. The purpose of the study is to identify culturally specific resilience and risk factors that affect children's well-being and to then use the information to guide the development of culturally based interventions.

The project was designed in partnership with the participating reservations and reserves. Prior to the application funding, the research team was invited to work on these reservations, and tribal resolutions were obtained. As part of our agreement to work together the researchers promised that participating reservations would be kept confidential in published reports. On each participating reservation, an advisory board was appointed by the tribal council. The advisory boards were responsible for advising on the handling of difficult personnel problems, questionnaire development, reading reports for respectful writing, and assuring that published reports protected the identity of the respondents and the culture. Upon advisory board approval of the questionnaires, the study procedures and questionnaires were submitted for review by the university Institutional Review Board for approval. All participating staff on the reservations were approved by the advisory board and were either tribal members or in two cases non-members who are spouses of tribal members. To ensure quality of data collection, all the interviewers underwent special training for conducting pencil-and-paper and computer-assisted personal interviewing for the diagnostic measures. The training included practice and feedback sessions. In addition, all of the interviewers completed a required human subjects protection training that emphasized the importance of confidentiality and taught procedures to maintain the confidentiality of data.

Each tribe provided a list of families of enrolled children aged 10-12 years who lived on or proximate to (within 50 miles) the reservation or reserve. We attempted to contact all families with a target child with in the specified age range. Families were recruited during a personal visit by a Native interviewer during which the project was explained to them. (Note: Two of the more than 40 interviewers were non-Indian spouses of enrolled tribal members who were well-known and accepted in their respective communities.) The families were then presented with a gift of wild rice and invited to participate. If they agreed to be interviewed each family member received $\$ 40$ for their time when the interviews were completed. The recruitment procedure resulted in an overall response rate of $79.4 \%$. In this type of research where multiple fam- 
ilies must agree to participate, a response rate of $70 \%$ is usually viewed as acceptable.

\section{Sample characteristics}

The sample for this analysis was made up of 861 parents/caretakers (236 males and 625 females). Fathers/male caretakers ranged in age from 21 years to 68 years with an average age of 41 years; mothers/female caretakers ranged in age from 17 years to 77 years with an average of 39 years.

About one-third (36\%) of the families contained two biological parents and $23 \%$ were single mother-headed households. The remaining families were of various configurations: mother-stepfather, $10 \%$; mother living with other relatives (e.g., grandmothers, aunts, uncles) $7 \%$; single biological fathers, $4 \%$; child living with grandparents, $7 \%$; or other multigenerational household configurations.

The distribution of income in this sample varies according to family structure. Single-parent households were twice likely as two-parent households (includes stepfathers and live-ins) to have incomes of $\$ 15,000$ or less ( $46 \%$ vs. $23.5 \%$ ). More than one-fourth (27.9\%) of single-parent households were getting by on $\$ 10,000$ or less per year. Median income for single parent families was under $\$ 20,000$ compared to about $\$ 25,000$ for two-parent families. Financial assistance was also common. About one-half of both singleparents (53.5\%) and one-third of two-parent households $(34.7 \%)$ received food stamps. Approximately one-half (44\%) of single-parent households and $28.9 \%$ of two-parent households received family assistance (TANF) or the Canadian equivalent in the past year.

\section{Measurement}

The University of Michigan Composite International Diagnostic Interview (UM-CIDI) was used to assess five diagnoses: Alcohol abuse, alcohol dependence, drug abuse, major depressive disorder and generalized anxiety disorder. The UM-CIDI is based on Diagnostic and Statistical Manual-III-R (DSM-III-R) criteria and represents the University of Michigan revision of the CIDI [22] used in the National Comorbidity Survey (NCS) [6-8]. The CIDI, (WHO, 1990) from which the UM-CIDI is derived is a well-established diagnostic instrument [22] that has shown excellent inter-rater reliability, test-retest reliability, and validity for the diagnoses that were used in this study [23]. The UM-CIDI has been used extensively with trained interviewers who are not clinicians. The version used in this study included modifications similar to those in the AI-SUPERPFP $[5,21,24]$.

\section{Results}

\section{Lifetime and 12-month prevalence}

Three-fourths $(74.6 \%)$ of the American Indian and Canadian First Nations adults from this Northern Midwest culture met criteria for at least one of the five lifetime mental disorders (Table 1 ). Males $(85.6 \%)$ were more likely than females $(70.4 \%)$ to meet criteria for at least one lifetime disorder. About one-fourth (24.5\%) of the adults met criteria for two lifetime mental disorders and $7.1 \%$ met criteria for three or more of the five lifetime disorders. Approximately $25 \%$ of the adults met 12 month criteria for at least one of the five disorders, $6 \%$ met 12 -month criteria for two disorders, and 2.2\% met 12 -month criteria for three or more. The 12-month prevalence rates are substantially lower than lifetime rates reflecting earlier substance abuse behaviors or previous periods of depression or anxiety.

Substance abuse disorders were the most prevalent. Around $50 \%$ of the American Indian and Canadian First Nations adults met lifetime criteria for alcohol abuse with no significant difference between males and females (Table 2). Of all the adults $21 \%$ met lifetime criteria for alcohol dependence, with males $(28 \%)$ more likely than females $(18.2 \%)$ to have been dependent on alcohol at some point in their lives. Rates of 12-month alcohol dependence were much lower at approximately $4 \%$ with no significant differences between males and females.

A $22 \%$ of the American Indian and Canadian First Nations parents/caretakers met lifetime criteria for drug abuse. Males (28.8\%) were significantly more likely than females $(20 \%)$ to have met criteria for drug abuse at some point in their lives. About $8 \%$ of the respondents met 12-month criteria for drug abuse with no significant gender difference in 12-month prevalence.

Of the all respondents $17 \%$ met lifetime criteria for major depressive episode (MDE). Females $(20.0 \%)$ were twice as likely as males $(9.3 \%)$ to meet lifetime criteria for MDE. About $8 \%$ of the adults met 12 -month criteria for MDE with no significant gender difference in prevalence rates.

Very few $(4.5 \%)$ of the respondents met lifetime criteria for generalized anxiety disorder (GAD). Females $(5.6 \%)$ were about three times more likely than males $(1.7 \%)$ to meet lifetime criteria for GAD. Females $(3.7 \%)$ also were more likely than males $(0.8 \%)$ to meet 12 month criteria for GAD.

Residents from remote Canadian reserves were much less likely to meet lifetime criteria for alcohol abuse

Table 1. Lifetime and 12-month mental health disorder among Northern Midwest American Indian parents/caretakers of adolescents aged 10-12 years $^{\mathrm{a}}$

\begin{tabular}{|c|c|c|c|c|c|c|}
\hline & \multicolumn{2}{|l|}{ Total $(n=861)$} & \multicolumn{2}{|l|}{ Male $(n=236)$} & \multicolumn{2}{|c|}{ Female $(n=625)$} \\
\hline & Lifetime (\%) & 12-Month (\%) & Lifetime (\%) & 12-Month (\%) & Lifetime (\%) & 12-Month (\%) \\
\hline No disorder & 25.4 & 74.7 & $14.4^{* *}$ & 75.8 & 29.6 & 74.2 \\
\hline Two disorders & 24.5 & 6.4 & 26.7 & 5.9 & 23.7 & 6.6 \\
\hline Three and more disorders & 7.1 & 2.2 & 5.1 & 0.8 & 7.9 & 2.7 \\
\hline
\end{tabular}

**P $<0.05$ between males and females

a SAS V8 was used to obtain corrected standard errors and compare prevalence of mental disorders across gender. 
Table 2. Lifetime and 12-month prevalence of DSM-III-R-Disorders among Northern Midwest American Indian parents/ caretakers of adolescent aged 10-12 years

\begin{tabular}{|c|c|c|c|c|c|c|}
\hline & \multicolumn{2}{|c|}{ Total $(n=861)$} & \multicolumn{2}{|c|}{ Male $(n=236)$} & \multicolumn{2}{|c|}{ Female $(n=625)$} \\
\hline & Lifetime (\%) & 12-Month (\%) & Lifetime (\%) & 12-Month (\%) & Lifetime (\%) & 12-Month (\%) \\
\hline Alcohol abuse & $49.6(0.02)$ & $14.1(0.01)$ & $54.7(0.03)^{\mathrm{a}}$ & $12.7(0.02)$ & $47.7(0.02)$ & $14.6(0.01)$ \\
\hline Drug abuse & $22.4(0.01)$ & $7.9(0.01)$ & $28.8(0.03)^{*}$ & $10.2(0.02)$ & $20.0(0.02)$ & $7.0(0.01)$ \\
\hline Major depression & $17.1(0.01)$ & $8.1(0.01)$ & $9.3(0.02)^{* * *}$ & $5.5(0.01)$ & $20.0(0.02)$ & $9.1(0.01)$ \\
\hline Generalized anxiety & $4.5(0.01)$ & $2.9(0.02)$ & $1.7(0.01)^{* *}$ & $0.8(0.01)^{* *}$ & $5.6(0.01)$ & $3.7(0.01)$ \\
\hline
\end{tabular}

${ }^{*} P<0.05$ between males and females

${ }^{* *} P<0.01$ between males and females

${ }^{* * *} P<0.001$ between males and females

${ }^{a}$ Corrected standard errors are in parenthesis

(29.5\%) and drug abuse (2.6\%) than their rural U.S. reservation and non-remote Canadian reserve counterparts (49.6\% alcohol abuse; $22.4 \%$ drug abuse) (not shown). Rates of 12-month prevalence for alcohol abuse did not significantly differ by location of reservation or reserve. However, residents of remote reserves $(0.0 \%)$ were less likely than rural U.S. and non-remote reserve residents to meet 12 -month criteria for drug abuse $(8.7 \%)$. There were no significant remote versus non-remote differences in prevalence of other disorders.

\section{Comparison to adults in the National Comorbidity Survey} and the AI-SUPERPFP

To place the prevalence rates from this sample of Native parents/caretakers in perspective, rates for lifetime diagnoses were compared to national prevalence rates by selecting those in the same age categories (i.e., 17-54 years) from the U.S. population in the National
Comorbidity Survey (NCS) (Table 3). (Note: age range delimitation resulted in slight percentage changes for Northern Midwest prevalence rates from Table 2.) We also compared the rates for the Northern Midwest sample to those of AI-SUPERPFP (ages 15-54 years). To increase confidence in the comparisons, we used the AISUPERPFP DSM-III-R prevalence rates reported in the American Journal of Psychiatry (2005) [24] rather than the DSM-IV prevalence rates reported in the Archives of General Psychiatry (2005) [5]. Significance levels are not given in that our sample is a non-probability sample.

The American Indian and Canadian First Nations adult males in the Northern Midwest (54.7\%) were four times more likely to meet lifetime criteria for alcohol abuse than were NCS adults (13.1\%). (Note: Prevalence rates for alcohol abuse exclude those who meet criteria for alcohol dependence.) They were five times more likely to meet lifetime criteria for alcohol abuse than males from the AI-SUPERPFP Southwest cultures

Table 3. Comparison of Northern Midwest*, National Comorbidity Survey (NCS), \& AI-SUPERPFP lifetime diagnosis adult sample (age 17-54)**

\begin{tabular}{|c|c|c|c|c|c|c|c|c|}
\hline & \multirow{2}{*}{\multicolumn{2}{|c|}{ Northern Midwest }} & \multirow{2}{*}{\multicolumn{2}{|c|}{ NCS }} & \multicolumn{4}{|c|}{ AI-SUPERPFP } \\
\hline & & & & & \multicolumn{2}{|c|}{ Southwest Tribe } & \multicolumn{2}{|c|}{ Northern Plains } \\
\hline & $\%$ & $\mathrm{CI}$ & $\%$ & $\mathrm{CI}$ & $\%$ & $\mathrm{CI}$ & $\%$ & $\mathrm{CI}$ \\
\hline Alcohol abuse & 54.7 & $48.3-61.1$ & 13.1 & $11.9-14.1$ & 11.2 & $8.2-15.1$ & 12.8 & $9.9-16.3$ \\
\hline Alcohol dependence & 27.3 & $21.2-33.4$ & 22.6 & $21.2-23.9$ & 31.1 & $26.3-36.2$ & 30.5 & $26.2-35.2$ \\
\hline Drug abuse $\mathrm{e}^{* * *}$ & 32.1 & $25.7-38.4$ & 15.7 & $14.6-16.9$ & 5.3 & $3.4-8.4$ & 6.2 & $4.2-9.1$ \\
\hline Generalized anxiety & 1.9 & $1.9-3.4$ & 4.1 & $3.4-4.7$ & 3.2 & $1.8-5.7$ & 1.7 & $0.8-3.6$ \\
\hline \multicolumn{9}{|l|}{ Female } \\
\hline Alcohol abuse & 47.7 & $43.7-51.6$ & 7.2 & $6.4-7.9$ & 5.1 & $3.4-7.7$ & 10.3 & 7.7-13.5 \\
\hline Alcohol dependence & 19.1 & $15.9-22.4$ & 9.1 & $8.2-9.9$ & 8.7 & $6.4-11.7$ & 20.5 & $16.8-24.6$ \\
\hline Drug abuse $\mathrm{e}^{* * *}$ & 21.6 & $18.2-25.0$ & 8.6 & $7.8-9.5$ & 2.5 & $1.4-4.6$ & 4.5 & 2.9-7.1 \\
\hline Major depression & 20.7 & $17.4-24.1$ & 22.1 & 20.9-23.4 & 14.3 & 11.4-17.9 & 10.3 & 7.8-13.6 \\
\hline
\end{tabular}

*Numbers may not match Table 2 due to slight differences in age range

**Beals et al. (2005, pp. 1726-1727), Table $\underline{2}$

***Drug abuse rates for Northern Midwest may contain those who meet criteria for drug dependence and therefore are not directly comparable to the NCS or AI-SUPERPFP rates, which exclude those with drug dependence 
$(11.2 \%)$, and four times more likely than males from the Northern Plains cultures (12.8\%). Differences in rates of lifetime alcohol dependence were not as great. Around $27 \%$ of Northern Midwest American Indian and Canadian First Nations males met lifetime criteria for alcohol dependence compared to $22.6 \%$ of their NCS counterparts, $31.1 \%$ of AI-SUPERPFP Southwest males and $30.5 \%$ of Northern Plains males.

The comparisons of prevalence rates of drug abuse between the Northern Midwest sample, NCS and AISUPERPFP should be viewed with caution because the Northern Midwest rates may include those with drug dependence who were excluded in the drug abuse rates for the other two samples. This inflates prevalence rates for lifetime drug abuse in the Northern Midwest sample. However, even if we combine drug abuse and drug dependence rates for males from the AI-SUPERPFP Southwest culture $(14.9 \%$, not shown) and for males the Northern Plains cultures (15.7\%, not shown), the rate for drug abuse among males from the Northern Midwest sample $(32.1 \%)$ was substantially higher than either of the two AI-SUPERPFP samples.

Northern Midwest males reported lower rates of MDE $(9.6 \%)$ than did their counterparts in the NCS $(13.7 \%)$, but rates for MDE were very similar to Southwest $(9.8 \%)$ and Northern Plains $(7.2 \%)$ males in the AISUPERPFP study. The rate for GAD among the Northern Midwest males (1.9\%) was half that for NCS males $(4.1 \%)$, lower than that reported for Southwest males $(3.2 \%)$ and closely similar to that reported by Northern Plains males (1.7\%).

The prevalence of lifetime alcohol abuse among the Northern Midwest females was (47.7\%) more than six times that of their NCS counterparts (7.2\%), nine times that for AI-SUPERPFP Southwest females (5.1\%) and four times that for Northern Plains females (10.3\%). Although similar to women in the Northern Plains (20.5\%), Northern Midwest females (19.1\%) were twice as likely as NCS females $(9.1 \%)$ and Southwest females $(8.7 \%)$ to meet lifetime criteria for alcohol dependence.

As with Northern Midwest men, the women's prevalence rates for lifetime drug abuse may also include those who are drug dependent thus inflating the Northern Midwest rates. However, when the Northern Mid- west rates for lifetime drug abuse (21.6\%) are compared to the combined drug abuse and drug dependence rates for women in the AI-SUPERPFP sample in the Southwest $(4.2 \%$, not shown) or Northern Plains $(12.4 \%$, not shown), they are still substantially higher.

Rates of lifetime MDE among the Northern Midwest women $(20.7 \%)$ were very similar to those for NCS females (22.1\%). However, Northern Midwest women were more likely to meet lifetime criteria for MDE than Southwest $(14.3 \%)$ or Northern Plains women (10.3\%). The same was true for lifetime GAD. Northern Midwest females $(5.3 \%)$ were similar to NCS females $(6.4 \%)$, but reported higher rates of GAD than their Southwest $(3.6 \%)$ or Northern Plains (1.7\%) counterparts.

\section{Comorbidity}

Table 4 presents the lifetime rates for each disorder on the diagonal and comorbidity with other disorders below the diagonal. For example, of the $22.4 \%$ who met lifetime criteria for drug abuse, nearly all $(20.4 \%)$ were comorbid for lifetime alcohol abuse. Similarly, of those who met lifetime criteria for MDE and GAD, nearly all met criteria for alcohol abuse. Most adults who met criteria for GAD also met criteria for MDE.

\section{Multivariate analyses}

Logistic regression models were run to investigate correlates of mental disorders among Northern Midwest American Indian and Canadian First Nations parents/ caregivers (Table 5). In Model 1, being male and unmarried or divorced were associated with meeting criteria for any single lifetime mental disorder. Those residing on the remote Canadian reserves were less likely than those living on rural U.S. reservations and rural Canadian reserves to meet criteria for any single disorder. Males were about three times more likely than females to meet criteria for any of the five disorders. Unmarried or divorced persons were nearly twice as likely as married persons to meet criteria for one of the five disorders.

The likelihood of meeting lifetime criteria for alcohol abuse was investigated in Model 2. Being male, unmarried or divorced, and residing on a rural reservation or reserve rather than a remote reserve were associated

Table 4. Comorbidity among Northern Midwest American Indian parents/caretakers of adolescents aged 10-12 years (\%)

\begin{tabular}{|c|c|c|c|c|c|}
\hline & AA & $\mathrm{AD}$ & $\mathrm{DA}$ & GA & MD \\
\hline 1 Lifetime alcohol abuse & 49.6 & & & & \\
\hline 2 Lifetime alcohol dependence & $-*$ & 20.9 & & & \\
\hline 3 Lifetime drug abuse & 20.4 & 8.0 & 22.4 & & \\
\hline 4 Lifetime generalized anxiety & 3.8 & 1.4 & 1.7 & 4.5 & \\
\hline 5 Lifetime major depression & 15.1 & 6.7 & 5.3 & 3.0 & 17.1 \\
\hline
\end{tabular}


with meeting lifetime criteria for alcohol abuse. Meeting criteria for alcohol abuse was not associated with the likelihood of being comorbid with another of the five disorders.

The only significant correlate for meeting criteria for alcohol dependence (Model 3) was being male. However, meeting lifetime criteria for alcohol dependence increased the odds of meeting criteria for another of the five disorders more than four times.

Age was negatively associated with meeting lifetime criteria for drug abuse (Model 4). Being male and from rural reservations and reserves rather than remote Canadian reserves were also correlated with meeting lifetime criteria for this disorder. Family per capita income was negatively associated with meeting lifetime criteria for drug abuse. Meeting lifetime criteria for drug abuse increased the odds of being comorbid for one of the other five mental disorders more than five times.

The Northern Midwest females were more than four times more likely than their male counterparts to meet criteria for GAD and almost three times more likely than males to meet criteria for life time MDE (Models 5 and 6). Meeting criteria for GAD increased the odds of meeting criteria for another of the five disorders more than seven times; meeting criteria for MDE increased the odds of comorbidity more than five times.

\section{Discussion}

\section{Limitations}

An important caution in interpreting the results of this study is the sensitivity of UM-CIDI measures for assessing American Indian and Canadian First Nations people [3]. For example, drinking patterns among Native adults may include sporadic binge drinking at irregular intervals that may not be represented adequately by diagnostic criteria [25]. Also, the meaning and definitions of depression may vary across Native cultures [26]. However, this study uses the same measures as recent population studies of Native people to provide the best estimates possible.
Although we interviewed a broad range of Native adults on multiple reservations and Canadian reserves that are dispersed geographically across two Midwestern states and one Canadian province, these results pertain to a single culture and capture variations within this culture. We believe the findings represent the culture well, but they cannot be generalized to other Native cultures. However, if we are to obtain sound psychiatric epidemiological information pertaining to Native people, it will be necessary to proceed nation by nation with comparable measures.

Another caution regarding the sample is that it is made up of parents and caretakers of children aged 1012 years. This could reflect a selection bias in that parents/caretakers may be more likely to have never used substances, quit drinking and to have remained alcohol and drug free. Also, parents/caretakers may have better mental health than non-custodial unmarried or divorced adults or adults who have never had children. We believe this should result in a conservative bias for the Northern Midwest sample.

A final concern is that this report is limited to only five psychiatric diagnoses. This limitation was dictated by time, sensitivity of the nations to certain diagnostic questions (e.g., antisocial personality disorders, posttraumatic stress disorder), and the intent of the longitudinal study (i.e., risk and protective factors for Native children). We had to make difficult decisions based on tribal preferences and subject burden. For example, in our attempt to capture drug use histories of parents we have only rates for drug abuse and not drug dependence. This has the effect of inflating the prevalence rates of drug abuse.

\section{Comparisons with NCS and AI-SUPERPFT: cultural differ- ences or method variance?}

Prevalence rates diverged for four of the disorders. Lifetime rates for alcohol abuse were much higher among American Indian adults in the Northern Midwest than for the NCS and AI-SUPERPFP adults, particularly so among women. It should be noted that the lifetime al-

Table 5. Logistic regression model predicting lifetime mental disorder among Northern Midwest American parents/caretakers of adolescents aged 10-12 years

\begin{tabular}{|c|c|c|c|c|c|c|c|c|c|c|c|c|}
\hline & \multicolumn{2}{|c|}{$\begin{array}{c}\text { Any single } \\
\text { mental disorder }\end{array}$} & \multicolumn{2}{|c|}{$\begin{array}{c}\text { Alcohol } \\
\text { abuse }\end{array}$} & \multicolumn{2}{|c|}{$\begin{array}{c}\text { Alcohol } \\
\text { dependence }\end{array}$} & \multicolumn{2}{|c|}{ Drug abuse } & \multicolumn{2}{|c|}{$\begin{array}{c}\text { Generalized } \\
\text { anxiety }\end{array}$} & \multicolumn{2}{|c|}{$\begin{array}{c}\text { Major } \\
\text { depression }\end{array}$} \\
\hline & $b$ & $\operatorname{Exp}(b)$ & $b$ & $\operatorname{Exp}(b)$ & $b$ & $\operatorname{Exp}(b)$ & $b$ & $\operatorname{Exp}(b)$ & $b$ & $\operatorname{Exp}(b)$ & $b$ & $\operatorname{Exp}(b)$ \\
\hline Gender (female $=1$ ) & -1.07 & $0.34^{* *}$ & -0.38 & $0.68^{*}$ & -0.62 & $0.54^{* *}$ & -0.55 & $0.57^{* *}$ & 1.45 & $4.27^{* *}$ & 1.04 & $2.84^{* * *}$ \\
\hline Marital status (married $=1$ ) & -0.55 & $0.58^{* *}$ & -0.33 & $0.72 *$ & -0.21 & 0.8 & -0.1 & 0.91 & -0.17 & 0.84 & -0.19 & 0.83 \\
\hline Family income per capita in 1000 's & 0.01 & 1.01 & 0.01 & 0.99 & 0.01 & 1.01 & -0.05 & $0.95^{* *}$ & 0.02 & 1.02 & 0.01 & 1.01 \\
\hline Remote $($ remote $=1)$ & -1.3 & $0.27^{* *}$ & -0.98 & $0.38^{* *}$ & -0.21 & 0.81 & -2.4 & $0.09 * *$ & -0.95 & 0.39 & 0.06 & 1.06 \\
\hline Model $\chi^{2}$ & \multicolumn{2}{|c|}{$60.46 * *$} & \multicolumn{2}{|c|}{$25.10^{* * *}$} & \multicolumn{2}{|c|}{$80.21 * * *$} & \multicolumn{2}{|c|}{$116.64^{* * *}$} & \multicolumn{2}{|c|}{$24.52^{* * *}$} & \multicolumn{2}{|c|}{$61.13^{* * *}$} \\
\hline
\end{tabular}

${ }^{* * *} P<0.001 ;{ }^{* *} P<0.01 ; * P<0.05$ 
cohol abuse rates reported exclude those who met criteria for alcohol dependence. The rates for lifetime alcohol dependence were quite similar across cultures for male participants and for Northern Midwest and Northern Plains female participants. The variation in reported alcohol abuse and alcohol dependence may be indicative of different drinking patterns across the three cultures or it may reflect method variance.

The rates for lifetime drug abuse were higher among the Northern Midwest adults even when we compared them to the combined rates for drug abuse and drug dependence for the AI-SUPERPFP. Although the rates for lifetime MDE were similar across cultures for males, the Northern Midwest women reported much higher lifetime MDE than Southwest or Northern Plains women. There was a similar trend for GAD.

Given the numerous methodological considerations, how do we distinguish between actual cultural differences in prevalence and potential methods variance? First, it is important to evaluate the sampling procedures. Specialized or clinical samples should not be generalized to the culture as a whole. For example, the Northern Midwest sample is made up of adult caretakers of children aged 10-12 years. This sample should reflect a conservative bias of adults who are more likely to be married, employed and have children. The Northern Midwest sample does not reflect the substance abuse or mental health problems of those under 17 years, those older than 54 years, and others without children within the population. We attempted to decrease age variation in our comparisons to the NCS and AI-SUPERPFP samples by selecting those aged 17-54 years from the NCS and the Northern Midwest sample in our comparisons in Table 3. Those in the AI-SUPERPFP sample were aged $15-54$ years, a difference of only 2 years at the younger end of the age range.

Given what we believe is a conservative sampling bias, it is puzzling that Northern Midwest lifetime prevalence rates for some of the disorders are so much higher than those for the Southwest and Northern Plains cultures. One possibility is that the diagnostic interview schedule itself operates differently by American Indian cultures such that it may result in differences in prevalence rates by culture, systematically under estimating mental disorders in some cultures while over estimating them in others (e.g., alcohol abuse, drug abuse, MDE and GAD among women).

Another way to evaluate variance in prevalence rates across cultures is to consider previous research on symptom counts, social problems, and social contexts. For example, May and Gossage (2001) report that among individuals from four tribes in the north central and northern Rocky Mountain states aged 16 years and older, "Over $60 \%$ of all males under the age of 40 report drinking 5 or more drinks per occasion in the past 30 days. Among females in the same age groups, 39-45\% report drinking similarly large quantities" (p. 14) [16]. The rates for those over $40-49$ years drop to $49 \%$ of males and $25.4 \%$ of females. For those aged 50 and older, the rates are $33.9 \%$ of males and $14.7 \%$ of females. Though not strictly comparable, these rates of heavy drinking in the Northern Plains and Rocky Mountain tribes provide some evidence that the alcohol abuse rates of $54.7 \%$ for males and $47.7 \%$ for females in the Northern Midwest sample may be reasonable.

There is corroborating research that shows that some Southwest cultures have lower rates of substance abuse and mental health problems (e.g., suicide) than other American Indian cultures [27, 28]. However, there is also previous research that suggests high rates of social problems, substance abuse and mental health problems among some Northern Plains cultures [28, 29]. Given the numerous reports of social problems and alcohol use it is difficult to reconcile the low lifetime prevalence rates of alcohol abuse and drug abuse among the Northern Midwest and Northern Plains men and between alcohol, drug abuse, depression, and anxiety among the women in the three cultures.

If method variance accounts for the cultural differences it could be that flaws in the Northern Midwest sampling and interview procedures led to a symptom over count. However, although we can think of many reasons for the respondents to under report symptoms such as alcohol abuse or drug abuse, it is hard think of reasons for over reporting them. Conversely, there could be procedures in AI-SUPERPFP that resulted in a symptom under count in one or both cultures. It is also possible that the cultures sampled, particularly those in the Northern Plains were less likely to report depressive symptoms and substance abuse problems.

It also could be that interviewing practices resulted in divergent results. Standard Northern European methodological procedures used in AI-SUPERPFP [5] such as audio taping diagnostic interviews for fidelity (p. 100) may affect openness of the respondents who are already distrustful of research. In the Northern Midwest study, interviewers were from the same reservation as the respondents and the respondents had the option of selecting the interview team with whom they were most comfortable to avoid having friends or relatives for interviewers, contamination of reservation "feuds," and issues of trust surrounding confidentiality. Our concern was the familiarity of the interviewers would result in under reporting of symptoms.

\section{Treatment and prevention implications}

Regardless, of comparisons with other American Indian cultures, the rates of mental disorder among the Northern Midwest adults are a matter of concern. An $85 \%$ of 
the Northern Midwest men and $70.4 \%$ of the women met lifetime criteria for at least one of the five psychiatric disorders. Nearly, one-third met lifetime criteria for two or more disorders. All were caretakers of children aged 10-12 years. These findings have serious implications for effective parenting and family functioning. The prevalence rates also call attention to the need to improve access, identify and eliminate cultural barriers, and improve funding for mental health services among Northern Midwest and Canadian First Nations people.

The findings reflect unique patterns of psychiatric disorders, particularly the ubiquity of substance abuse disorders and their co-occurrence with internalizing disorders (e.g., MDE and GAD). Patterns of comorbidity indicate that substance abuse disorders and internalization disorders greatly overlap. Addressing one without identifying and treating the other weakens the chances of positive outcomes. Clearly, reducing lifetime occurrences of substance abuse disorders would have an enormous impact on the mental health of this population.

Even though consciousness has been rising for several decades concerning the need for culturally specific approaches to mental health and substance abuse services among Native people, funding agencies and training programs continue to lag behind "grass roots" community efforts to make these services culturally accessible. Too often, mental services continue to make the same mistakes they have for decades [30]. Some problems take time to be remedied such as the shortage of trained Native mental health services professionals, but these results indicate an urgent need to bring culturally accessible mental health services to American Indian and Canadian First Nations People.

\section{Future research}

There are very few psychiatric diagnostic studies of American Indian and Canadian First Nations people $[4,21]$ and those that exist often have not used the same structured psychiatric diagnostic interview schedules [3]. AI-SUPERPFP has mapped the terrain [5, 24]. However, we need additional culturally sensitive epidemiological studies that progress nation by nation to build a coherent picture of the psychological well-being of American Indian, Alaska Native, and Canadian First Nations people and then systematic research programs that identify risk, resilience, and barriers to services utilization on which to build good practices.

But there are methodological problems that need attention first. We need to systematically address methodological issues so that we can disentangle differences attributable to cultural context and those attributable to methods variance. This will involve organized comparisons of procedures and measures for epidemiological research among Native people with goal of uniformity in procedures and measures that will result in cumulative knowledge. It will mean identifying and addressing procedures that could result in over or under reports of symptoms. Moreover, if the current findings hold up and reflect actual cultural variations in mental disorders, future research needs to address the historical and contextual differences that may account for difference in risk and protective factors across American Indian cultures.

\section{References}

1. Snipp M (1996) The size and distribution of the American Indian population: fertility, mortality, residence and migration. In: Sandefur G, Rindfuss R, Cohen B (eds) Changing numbers, changing needs: American Indian demography and public health. National Academy Press, Washington, DC, pp 17-52

2. US Department of the Interior, National Park Service. National NAGPRA: Indian Reservations in the Continental United States Map Index [Online]. Available at: http:// www.cr.nps.gov/nagpra/DOCUMENT/ResMapIndex. HTM. Accessed June 22, 2005

3. Beals J, Manson SM, Mitchell C, Spicer P, AI-SUPERPFP Team (2003) Cultural specificity and comparison in psychiatric epidemiology: walking the tightrope in American Indian Research. Cult Med Psychol 27:259-289

4. Duran B, Sanders M, Skipper B, et al. (2004) Prevalence and correlates of mental disorders among Native American women in primary care. Am J Public Health 94:71-77

5. Beals J, Manson SM, Whitesell NR, Spicer P, Novins DK, Mitchell DM, AI-SUPERPFP Team (2005) Prevalence of DSM-IV Disorders and attendant help-seeking in 2 American Indian reservation populations. Arch General Psychiat 62:99-108

6. Kessler RC (1994) The National Comorbidity Survey of the United States. Int Rev Psychiat 6:365-376

7. Kessler RC (1994) Building on the ECA: The National Comorbidity Survey and the children's ECA. Int J Method Psychiat Res 4:81-94

8. Wittchen HU, Kessler RC (1994) Modifications of the CIDI in the National Comorbidity Study: the development of the UM-CIDI. NCS Working Paper \# 2, Ann Arbor, MI

9. Kinzie J, Leung P, Boehnlein J et al. (1992) Psychiatric epidemiology of an Indian village: a 19-year replication study. J Nerv Ment Dis 180:33-39

10. Shore J, Kinzie J, Hampson J, Pattison E (1973) Psychiatric epidemiology of an Indian village. Pyschiatry 36:70-81

11. Beals J, Piasecki J, Nelson S, et al. (1997) Psychiatric disorder among American Indian adolescents; prevalence in Northern Plains youth. J Am Acad Child Adol Psychiat 36:1252-1259

12. Costello E, Angold A, Burns B, et al. (1996) The Great Smokey Mountain Study of youth: goals, design, methods, and the prevalence of DSM-III-R disorders. Arch General Psychiat 53:1129-1136

13. Costello E, Farmer M, Angold A, Burns B, Erklani A (1997) Psychiatric disorders among American Indian and white youth in Appalachia: the Great Smokey Mountains Study. Am J Public Health 87:827-832 
14. Kunitz S, Levy J (2000) Drinking, conduct disorder, and social change: Navajo experiences. Oxford University Press, New York

15. Leuning P, Kinzie J, Boehnlein J, Shore J (1993) A prospective study of the natural course of alcoholism in a Native American village. J Stud Alcohol 54:733-738

16. May P, Gossage J (2001) New data on the epidemiology of adult drinking and substance use among American Indians of the northern states: male and female data on prevalence, patterns, and consequences. Am Indian Alaska Native Mental Health Res 10:1-26

17. Beals J, Manson S, Shore J, et al. (2002) The prevalence of posttraumatic stress disorder among American Indian Vietnam veterans: disparities, and context. J Trauma Stress 15:89-97

18. Manson S (2000) Mental health services for American Indians and Alaska Natives: need, use, and barriers to effective care. Can J Psychiat 45:617-626

19. Indian Health Service (1990) National Plan for Native American Mental Health Services. U.S. Dept. of Health and Human Services, Rockville, MD

20. Wilson C, Civic D, Glass D (1995) Prevalence and correlate of depressive syndromes among adults visiting an Indian Health Service primary care clinic. Am Indian Alaska Native Mental Health Res 6:1-12

21. Spicer P, Beals J, Croy C, et al. (2003) The prevalence of DSM-III-R alcohol dependence in two American Indian populations. Alcohol Clin Exp Res 27:1785-1797

22. World Health Organization (1990) Composite International Diagnostic Interview (CIDI). Version 1.0. World Health Organization, Geneva
23. Wittchen HU (1994) Reliability and validity studies of the Who-Composite International Diagnostic Interview (CIDI): a critical review. J Psychiat Res 28:57-84

24. Beals J, Novins D, Whitesell N, et al. (2005) Prevalence of mental disorders and utilization of mental health services in two American Indian reservation populations: mental health disparities in a national context. Am J Psychiat 162:1723-1732

25. May P (1996) Overview of alcohol abuse epidemiology for American Indian populations. In: Sandefur G, Rindfuss R, Cohen $B$ (eds) Changing numbers, changing needs: American Indian demography and public health. National Academy Press, Washington, DC, pp 235-261

26. Manson S (1995) Culture and major depression: current changes in the diagnosis of mood disorders. Cult Psychiat 487-501

27. Novins DK, Beals J, Roberts RE, Manson SM (1999) Factors associated with suicide ideation among American Indian adolescents: does culture matter? Suicide Life Threat Behav 29(4):332-346

28. May PA (1987) Suicide and self-destruction among American Indian youths. Am Indian Alaska Native Mental Health Res 1:52-69

29. Zitzow D, Desjarlait F (1994) A study of suicide attempts comparing adolescents to adults on a northern plains American Indian reservation. Am Indian Alaska Native Mental Health Res 4(mono):35-69

30. Walls M, Johnson K, Whitbeck L, Hoyt D (in press) Mental health and substance abuse services preferences among American Indian people of the northern Midwest. Commun Mental Health J 